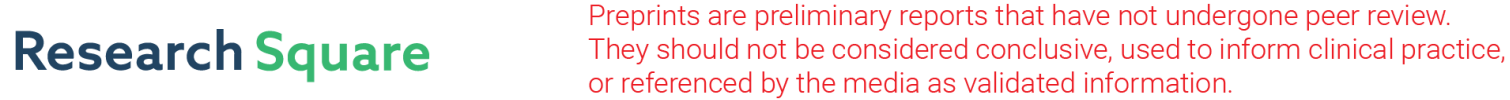 \\ Ground-state cooling of mechanical resonators by hot thermal light
}

Muhammad Naseem ( $\nabla$ mnaseem16@ku.edu.tr)

Koç University

Özgür Müstecaplıoğlu

Koç University https://orcid.org/0000-0002-9134-3951

Article

Keywords:

Posted Date: December 7th, 2020

DOI: https://doi.org/10.21203/rs.3.rs-111621/v1

License: (c) (i) This work is licensed under a Creative Commons Attribution 4.0 International License. Read Full License

Version of Record: A version of this preprint was published at Communications Physics on May 12th, 2021. See the published version at https://doi.org/10.1038/s42005-021-00599-z. 


\title{
Ground-state cooling of mechanical resonators by hot thermal light
}

\author{
M. Tahir Naseem ${ }^{1}$ and Özgür E. Müstecaplığ $\operatorname{lu}^{1, *}$ \\ ${ }^{1}$ Department of Physics, Koç University, 34450 Sariyer, Istanbul Turkey
}

(Dated: November 19, 2020)

\begin{abstract}
We propose a scheme to cool down a mechanical resonator to its quantum ground-state, which is interacting with a cavity mode via the optomechanical coupling. As opposed to standard laser cooling schemes where coherence renders the state of the resonator to its ground-state, here we use incoherent thermal light to achieve the same aim. We show that simultaneous cooling of two degenerate or near-degenerate mechanical resonators is possible in our scheme, which is otherwise a challenging goal to achieve in optomechanics. The generalization of this method to the simultaneous cooling of multiple resonators is straightforward. The underlying physical mechanism of cooling is explained by revealing a direct connection between the laser sideband cooling and "cooling by heating" in a standard optomechanical setting.
\end{abstract}

\section{INTRODUCTION}

The ground-state cooling of mechanical resonators is crucial for various applications, such as gravitational wave detection [1], ultrasensitive precision measurements [2], and for the investigation of the quantum behavior of mechanical systems [3], where only relevant motion is due to zero-point fluctuations. For the quantum information processing applications of optomechanical systems, mechanical resonators need to be close to their quantum ground-state $[4,5]$. To achieve this goal, different methods have been proposed and realized such as resolved sideband cooling [6-13], feedback-assisted cooling [14-19], and backaction cooling [20, 21]. In a standard approach, thermal energy is removed from a single mode of mechanical resonator to bring its state near to the ground-state. In the recent past, optomechanical systems having multiple mechanical resonators caught much attention [22-26], finding various applications such as heat management [27, 28] and mesoscopic entanglement [29]. However, ground-state cooling of degenerate or near-degenerate multiple mechanical resonators is quite a challenging task to accomplish in optomechanics. This is because the existence of dark modes in multiple resonators case, which decouple from the common cavity mode and hinders the simultaneous ground-state cooling of multiple resonators [18, 30-32]. There are few recent proposals $[33,34]$ to overcome this difficulty, however, these demand phase-dependent coherent interaction between the mechanical resonators.

A complementary approach, namely "cooling by heating", exploits incoherent driving of the quantum system to cool it down $[35,36]$. As opposed to laser cooling, in this method, incoherent thermal drive removes energy from the quantum system only to dump it in another bath at a lower temperature. For optomechanical setting, this has been proposed for two optical modes coupled to a single mechanical resonator [35]. This scheme requires not only three-body interaction but also it can not cool down the mechanical resonator near to its groundstate for the typical optomechanical system parameters.

In this work, we propose a scheme based on cooling by heating mechanism, and it requires only two-body interaction

*omustecap@ku.edu.tr to cool a single mechanical resonator to its ground-state. In addition, simultaneous ground-state cooling of multiple degenerate or near-degenerate mechanical resonators is also possible, which is otherwise challenging to accomplish. A key to realize this method is bath spectral filtering (BSF) [37-44], in which unwanted frequencies are filtered out from the systembath coupling spectra. The BSF has previously been shown to enhance performance of different thermal functions [43, 44]. Finally, we reveal a direct connection between the laser sideband cooling and cooling by heating in the optomechanical setting, which also explains the underlying physical mechanism of cooling in our scheme.

This paper is organized as follows. In Sec. II, we present our basic model and outline the derivation of the master equation. In Sec. III, we discuss the results of single and multiple resonators cooling. We present the conclusions of our work in Sec. IV.

\section{DESCRIPTION OF THE MODEL}

We consider a multimode optomechanical system (Fig. 1), which consists of a single optical mode interacting with two mechanical resonators. The optical mode is coupled to two thermal baths at temperatures $T_{c}$ and $T_{h}$, and each mechanical resonator of frequency $\omega_{i}$ is in contact with an independent thermal bath at temperature $T_{i}, i=1,2$. The Hamiltonian of the isolated optomechanical system is given by (we take $\hbar=1$ )

$$
\hat{H}_{s}=\omega_{a} \hat{a}^{\dagger} \hat{a}+\sum_{i=1}^{2}\left[\omega_{i} \hat{b}_{i}^{\dagger} \hat{b}_{i}-g_{i} \hat{a}^{\dagger} \hat{a}\left(\hat{b}_{i}+\hat{b}_{i}^{\dagger}\right)\right]
$$

where $\omega_{a}$ and $\omega_{i}$ are the frequencies of the optical and mechanical resonators, respectively. The bosonic annihilation (creation) operators of the optical and mechanical modes are given by $\hat{a}\left(\hat{a}^{\dagger}\right)$ and $\hat{b}_{i}\left(\hat{b}_{i}^{\dagger}\right)$, respectively. The $g_{i}$ terms represent the single-photon optomechanical coupling between the optical mode and mechanical resonators. This multimode optomechanical model can be realized via a photonic crystal optomechanical system [45] or circuit electromechanical system $[46,47]$. The free Hamiltonians of the independent ther- 
mal baths is given by

$$
\hat{H}_{B x}=\sum_{k, x} \omega_{k, x} \hat{a}_{k, x}^{\dagger} \hat{a}_{k, x}
$$

where, $x=H, C, i$ represents the hot, cold, and mechanical baths, respectively. In addition, the infinite number of bath modes are indexed by $k$. We need to attach two baths with the cavity mode, because the hot bath removes energy from the mechanical resonator and dumps it in a bath at lower temperature. In our scheme, cooling with the single optical bath is prohibited by the second law of thermodynamics (cf. Fig. 2). The interaction of the isolated optomechanical system with the thermal baths is given by the Hamiltonian

$$
\begin{aligned}
\hat{H}_{S B}=\sum_{k} g_{k, \alpha}\left(\hat{a}+\hat{a}^{\dagger}\right) \otimes\left(\hat{a}_{k, \alpha}+\hat{a}_{k, \alpha}^{\dagger}\right) & \\
& +\sum_{k, i} g_{k, i}\left(\hat{b}_{i}+\hat{b}_{i}^{\dagger}\right) \otimes\left(\hat{a}_{k, i}+\hat{a}_{k, i}^{\dagger}\right),
\end{aligned}
$$

where, $\alpha=H, C$, and the first term describes the interaction of hot and cold baths with the optical mode, and the second term represents the interaction between the mechanical resonator $b_{i}$ and its bath $B_{i}$.

To derive the master equation, we diagonalize the Hamiltonian of the isolated optomechanical system by a unitary transformation

$$
\hat{S}=e^{-\sum_{i} \zeta_{i} \hat{a}^{\hat{a}} \hat{a}\left(\hat{b}_{i}^{+}-\hat{b}_{i}\right),}
$$

where $\zeta_{i}=g_{i} / \omega_{i}$. The diagonalized Hamiltonian reads as

$$
\tilde{H}_{s}=\omega_{a} \tilde{a}^{\dagger} \tilde{a}+\sum_{i}\left[\omega_{i} \tilde{b}_{i}^{+} \tilde{b}_{i}-\frac{g_{i}^{2}}{\omega_{i}}\left(\tilde{a}^{\dagger} \tilde{a}\right)^{2}\right]
$$

and the transformed operators are given by

$$
\begin{aligned}
\tilde{a} & =\hat{S} \hat{a} \hat{S}^{+}=\hat{a} e^{-\sum_{i} \zeta_{i}\left(\hat{b}_{i}^{\dagger}-\hat{b}_{i}\right)}, \\
\tilde{b}_{i} & =\hat{S} \hat{b}_{i} \hat{S}^{\dagger}=\hat{b}_{i}-\zeta_{i} \hat{a}^{\dagger} \hat{a} .
\end{aligned}
$$

The eigenenergies of the isolated optomechanical system are expressed as

$$
E_{n_{a}, m_{i}}=n_{a} \omega_{a}+m_{i} \omega_{i}-n_{a}^{2} \frac{g_{i}^{2}}{\omega_{i}}
$$

where $m_{i}$ is the number of phonons in the mechanical resonator of frequency $\omega_{i}$, and $n_{a}$ is the number of photons in the cavity. The master equation in the interaction picture evaluate to

$$
\dot{\tilde{\rho}}=\tilde{\mathcal{L}}_{H}+\tilde{\mathcal{L}}_{C}+\tilde{\mathcal{L}}_{i},
$$

where the interaction of the baths with the system is described by Liouville superoperators $\tilde{\mathcal{L}}_{x}$, which are given by $[43,44$, 48]

$$
\begin{aligned}
\tilde{\mathcal{L}}_{\alpha} & =G_{\alpha}\left(\omega_{a}\right) \tilde{\mathcal{D}}[\tilde{a}]+G_{\alpha}\left(-\omega_{a}\right) \tilde{\mathcal{D}}\left[\tilde{a}^{\dagger}\right] \\
& +\sum_{i} \zeta_{i}^{2}\left[G_{\alpha}\left(\omega_{-}\right) \tilde{\mathcal{D}}\left[\tilde{a} \tilde{b}_{i}^{\dagger}\right]+G_{\alpha}\left(-\omega_{-}\right) \tilde{\mathcal{D}}\left[\tilde{a}^{\dagger} \tilde{b}_{i}\right]\right.
\end{aligned}
$$

(a)

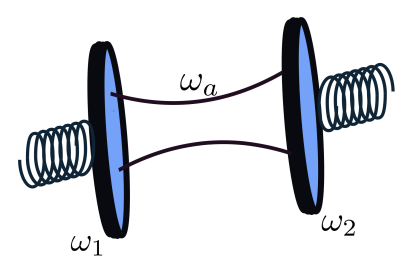

(c)

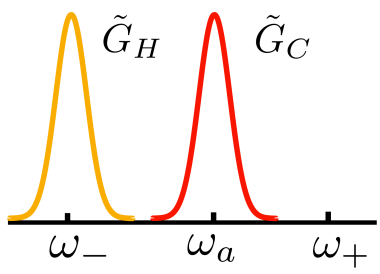

(b)

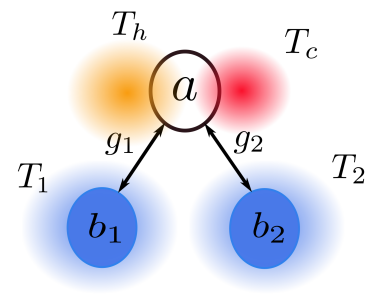

$(d)$

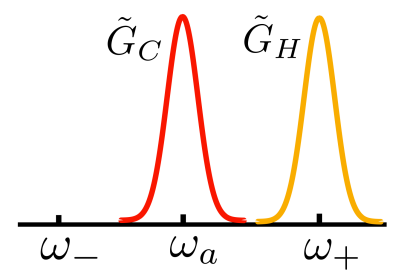

FIG. 1. (Color online) (a) Schematic illustration of the optomechanical system being considered in this work. A single optical mode of frequency $\omega_{a}$ is coupled to two mechanical resonators of frequencies $\omega_{1}$ and $\omega_{2}$ via optomechanical couplings $g_{1}$ and $g_{2}$, respectively. (b) Each mechanical resonator $\left(b_{i}\right)$ of frequency $\omega_{i}$ is in contact with an independent thermal bath at temperature $T_{i}, i=1,2$. In addition, the optical mode is in contact with two independent thermal baths at temperatures $T_{h}$ and $T_{c}$. (c) and (d) filtered spectra of the optical hot and cold baths given by Eq. (18), for cooling and heating process, respectively.

$$
\begin{aligned}
& \left.+G_{\alpha}\left(\omega_{+}\right) \tilde{\mathcal{D}}\left[\tilde{a} \tilde{b}_{i}\right]+G_{\alpha}\left(-\omega_{+}\right) \tilde{\mathcal{D}}\left[\tilde{a}^{+} \tilde{b}_{i}^{\dagger}\right]\right], \\
\tilde{\mathcal{L}}_{i} & =G_{i}\left(\omega_{i}\right) \tilde{\mathcal{D}}\left[\tilde{b}_{i}\right]+G_{i}\left(-\omega_{i}\right) \tilde{\mathcal{D}}\left[\tilde{b}_{i}^{\dagger}\right]
\end{aligned}
$$

where $\omega_{ \pm}=\omega_{a} \pm \omega_{b}$, and the Lindblad dissipators $\tilde{D}[\tilde{o}]$ are defined as

$$
\tilde{\mathcal{D}}[\tilde{o}]=\frac{1}{2}\left(2 \tilde{o} \tilde{\rho} \tilde{o}^{\dagger}-\tilde{o}^{\dagger} \tilde{o} \tilde{\rho}-\tilde{\rho} \tilde{o}^{\dagger} \tilde{o}\right),
$$

and the bath coupling spectrum has the form (we take $\mathrm{k}_{\mathrm{B}}=1$ )

$$
G_{x}(\omega)=\int_{-\infty}^{\infty} e^{i \omega t}\left\langle B_{x}(t) B_{x}(0)\right\rangle d t=e^{\omega / T_{x}} G_{x}(-\omega) .
$$

Note that, in Eq. (10), we have considered the weak optomechanical coupling regime [40], i.e.,

$$
\zeta_{i}^{2}\left\langle\tilde{b}_{i}^{\dagger} \tilde{b}_{i}\right\rangle \ll 1,
$$

and ignored all higher order terms $\mathcal{O}\left(\zeta^{3}\right)$.

\section{RESULTS}

In this section, we present the results for the ground-state cooling of the multiple mechanical resonators in an optomechanical system. To explain the underlying physical mechanism, first, we will consider a single mechanical resonator. 
This simple model helps us to make a direct connection between the "cooling by heating" with laser sideband cooling obvious in the optomechanical system.

For the numerical simulations, we have considered parameters of a typical optomechanical system [49]: $\omega_{i}=2 \pi \times 10$ $\mathrm{MHz}, g_{i}=2 \pi \times 100 \mathrm{kHz}, \kappa_{i}=2 \pi \times 100 \mathrm{~Hz}, \kappa_{h}=\kappa_{c}=$ $2 \pi \times \mathrm{MHz}$. Initial average phonons in the mechanical resonator is $\bar{n}_{i}(0) \sim 2 \times 10^{3}$, and the corresponding temperature becomes $T_{i} \sim 1.5 \mathrm{~K}$. We take the temperature of the cold bath $T_{\mathcal{C}} \sim \mathrm{mK}$, while the temperature of the hot bath is larger by many order of magnitude, i.e., $4 \times 10^{4} \lesssim T_{h} \lesssim 4 \times 10^{7}$ $\mathrm{K}$. In the results, we have scaled all these parameters with the optical frequency $\omega_{a}$.

\section{A. Single mechanical resonator}

In the case of a single mechanical resonator, the index $i$ can be dropped in Eq. (9). We are interested in the dynamics of the mechanical resonator, therefore, upon taking trace over the optical mode, Eq. (9) takes the form

$$
\frac{d \tilde{\rho}}{d t}=\left(A^{-}+A_{\mathrm{th}}^{-}\right) \tilde{\mathcal{D}}\left[\tilde{b}_{1}\right]+\left(A^{+}+A_{\mathrm{th}}^{+}\right) \tilde{\mathcal{D}}\left[\tilde{b}_{1}^{\dagger}\right],
$$

here $A^{-}\left(A_{\mathrm{th}}^{-}\right)$is downward transition rate, and $A^{+}\left(A_{\mathrm{th}}^{+}\right)$is upward transition rate in the mechanical resonator due to the optical (mechanical) baths, and these are defined as

$$
\begin{aligned}
& A^{-}:=\zeta_{1}^{2} \sum_{\alpha=H, C}\left(G_{\alpha}\left(\omega_{+}\right)\left\langle\tilde{n}_{a}\right\rangle+G_{\alpha}\left(-\omega_{-}\right)\left\langle\tilde{n}_{a}+1\right\rangle\right) \\
& A^{+}:=\zeta_{1}^{2} \sum_{\alpha=H, C}\left(G_{\alpha}\left(\omega_{-}\right)\left\langle\tilde{n}_{a}\right\rangle+G_{\alpha}\left(-\omega_{+}\right)\left\langle\tilde{n}_{a}+1\right\rangle\right) \\
& A_{\mathrm{th}}^{-}:=G_{1}\left(\omega_{1}\right), \quad A_{\mathrm{th}}^{+}:=G_{1}\left(-\omega_{1}\right) .
\end{aligned}
$$

Note that we use $A^{ \pm}$for the transition rates to compare our results with the sideband cooling in the atomic and optomechanical systems [49].

In the rest of the work, we consider one dimensional Ohmic spectral densities of all the baths, given by [38-40]

$$
G_{x}(\omega)= \begin{cases}\kappa_{x}(\omega)\left[1+\bar{n}_{x}(\omega)\right] & \omega>0, \\ \kappa_{x}(|\omega|) \bar{n}_{x}(|\omega|) & \omega<0\end{cases}
$$

here, $\kappa_{x}$ is the system-bath coupling strength, and $\bar{n}_{x}(\omega)=$ $1 /\left[e^{\left(\omega / T_{x}\right)}-1\right]$ is the mean number of quanta in the respective bath. For the cooling of mechanical resonator, bath spectral filtering is applied only to optical baths, which yields $[37,44]$

$$
\tilde{G}_{\alpha}:=\frac{1}{\pi} \frac{\pi G_{\alpha}(\omega)}{\left[\left(\omega-\left(\omega(n+1 / 2)+\Delta_{\alpha}(\omega)\right)^{2}+\left(\pi G_{\alpha}(\omega)\right)^{2}\right]\right.},
$$

here $\Delta_{\alpha}(\omega)$ is the Lamb-shift induced by the thermal bath.

Cooling: in a typical optomechanical system, resolvedsideband $\left(\omega_{1}>\kappa_{h, c}\right)$ cooling can be induced if the input laser light of frequency $\omega_{L}$ is resonant with the lower sideband $\omega_{\mathrm{L}}=\omega_{-}=\omega_{a}-\omega_{1}[12,49]$. In this case, the
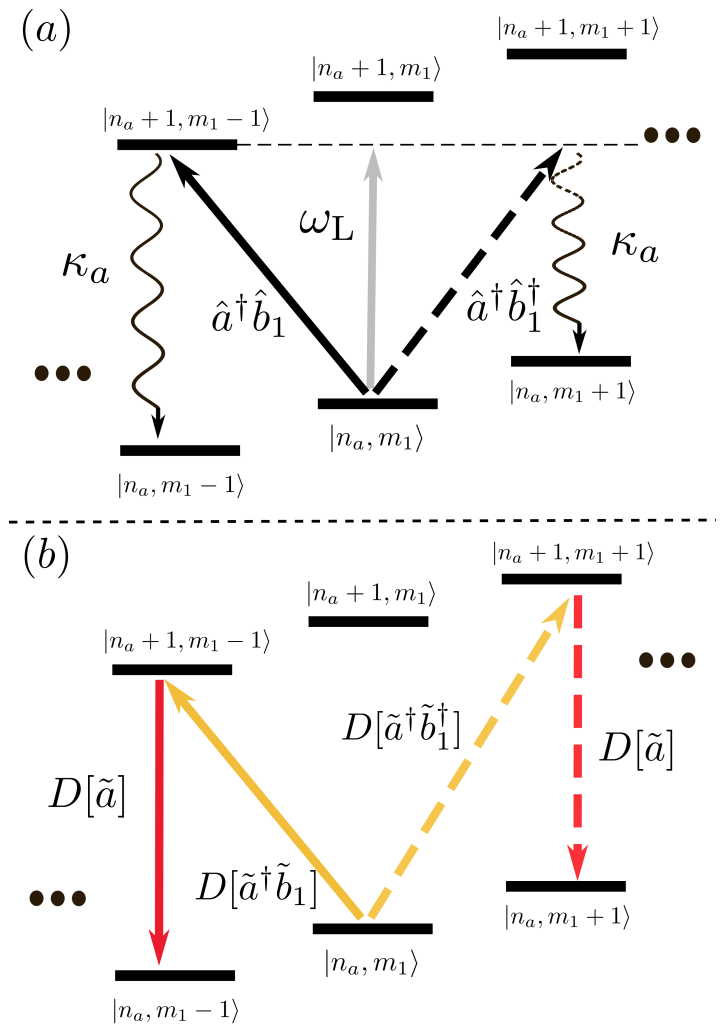

FIG. 2. (Color online) The underlying energy transitions between the dressed states $\left|n_{a}, m_{1}\right\rangle$ of the optomechanical system with a single mechanical resonator. In both panels, solid and dashed lines show the cooling and heating process, respectively. (a) The cooling and heating processes in the presence of coherent laser drive of frequency $\omega_{\mathrm{L}}$. The cooling process proceeds by the absorption on the lower sideband, leading to the leakage of a blue-shifted photon from the cavity by destroying one phonon. On contrary, absorption at the upper sideband leads to the heating of the mechanical resonator [10]. (b) The energy transitions involved in the cooling and heating process in our proposed scheme. As opposed to laser cooling, the transition at lower sideband $\left(\omega_{-}\right)$is induced by the hot bath via the incoherent system-bath interaction, given by $D\left[\tilde{a} \tilde{b}_{1}^{\dagger}\right]$ in Eq. (10). This creates an energetic (blue-shifted) photon which is dumped in a bath at lower temperature $T_{c}$. Cooling with a single optical bath is not allowed by the second law of thermodyanics. Because, in the case of a single optical bath, $\left|n_{a}, m_{1}\right\rangle \rightarrow\left|n_{a}+1, m_{1}-1\right\rangle \rightarrow\left|n_{a}, m_{1}-1\right\rangle$ transitions mean heat flows from the mechanical to optical bath for $T_{1}<T_{h}$, hence we need another bath to be consistent with the second law of thermodynamics. In the heating process, the hot bath induce the transition of frequency $\omega_{+}$following by dumping of a photon in the cold bath, which results in heating of the mechanical resonator. For the cooling process to dominate, the upper sideband frequency $\omega_{+}$is filtered from the system-bath coupling spectra, as shown in Fig. 1(c).

Stokes process is suppressed and anti-Stokes scattering dominates, as shown in Fig. 2(a). As long as the upper sideband $\omega_{+}=\omega_{a}+\omega_{1}$ is far off-resonant, the input laser can drive the mechanical resonator near to its ground-state. Compared with the standard optomechanical setup, we don't have input laser drive contribution in the Hamiltonian given in Eq. (1). Instead, the cavity is driven by the hot and cold thermal baths, however, both lower and upper phonon sidebands appear as a 
result of these thermal drives. By analogy with laser sideband cooling, we may want to drive the cavity at the lower sideband and suppress the upper sideband. This can be achieved by using bath spectral filtering [37-44].

To drive the cavity at lower sideband with the hot thermal bath, the filtered hot bath couples only to a transition frequency at $\omega_{-}$(lower sideband), and the coupling frequency at upper sideband $\omega_{+}$is filtered (Fig. 1(c)). To complete the refrigeration process, the cold bath couples to a transition frequency at $\omega_{a}$. The coupling spectra of the hot and cold baths are filtered and well-separated (Fig. 1(c)), and satisfy

$$
\begin{aligned}
\tilde{G}_{C}\left(\omega_{a}\right) & \gg \tilde{G}_{H}\left(\omega_{a}\right), \\
\tilde{G}_{C}\left(\omega_{-}\right) & \ll \tilde{G}_{H}\left(\omega_{-}\right) .
\end{aligned}
$$

For the choice of this bath spectral filtering, the master equation given in Eq. (9) modifies to

$$
\begin{aligned}
\tilde{\mathcal{L}}_{C} & =\tilde{G}_{C}\left(\omega_{a}\right) \tilde{\mathcal{D}}[\tilde{a}]+\tilde{G}_{C}\left(-\omega_{a}\right) \tilde{\mathcal{D}}\left[\tilde{a}^{\dagger}\right], \\
\tilde{\mathcal{L}}_{H} & =\zeta_{1}^{2}\left(\tilde{G}_{H}\left(\omega_{-}\right) \tilde{\mathcal{D}}\left[\tilde{a} \tilde{b}_{1}^{\dagger}\right]+\tilde{G}_{H}\left(-\omega_{-}\right) \tilde{\mathcal{D}}\left[\tilde{a}^{\dagger} \tilde{b}_{1}\right]\right), \\
\tilde{\mathcal{L}}_{1} & =G_{1}\left(\omega_{1}\right) \tilde{\mathcal{D}}\left[\tilde{b}_{1}\right]+G_{1}\left(-\omega_{1}\right) \tilde{\mathcal{D}}\left[\tilde{b}_{1}^{\dagger}\right]
\end{aligned}
$$

we note that $\tilde{\mathcal{L}}_{1}$ remains the same. The optical mode reaches to almost a thermal steady-state at temperature $T_{C}$ with corrections of the order $\mathcal{O}\left(\zeta^{2}\right)[40,41,50]$, and contribution from the mechanical bath. In our results, we ignore the correction term of the order $\zeta^{2}$ from the steady-state mean photon number $\left\langle\tilde{n}_{a}\right\rangle_{\mathrm{ss}}$, which then takes the form

$$
\left\langle\tilde{n}_{a}\right\rangle_{\mathrm{ss}} \approx \frac{\tilde{G}_{C}\left(-\omega_{a}\right)+\tilde{G}_{1}\left(-\omega_{1}\right)}{\left(\tilde{G}_{C}\left(\omega_{a}\right)+\tilde{G}_{C}\left(-\omega_{a}\right)\right)+\left(\tilde{G}_{1}\left(\omega_{1}\right)+\tilde{G}_{1}\left(-\omega_{1}\right)\right)} .
$$

The comparison between the numerical simulation of the master equation given in Eq. (20), and our approximate results is given in Appendix A (Fig 5). From Eq. (20), one can obtain the mean number of phonons in the mechanical resonator

$$
\frac{d\left\langle\tilde{n}_{1}\right\rangle}{d t}=\left(A_{c}^{+}+A_{\mathrm{th}}^{+}\right)\left\langle\tilde{n}_{1}+1\right\rangle-\left(A_{c}^{-}+A_{\mathrm{th}}^{-}\right)\left\langle\tilde{n}_{1}\right\rangle,
$$

here $A_{c}^{+}\left(A_{c}^{-}\right)$represents upward (downward) transition rate due to the optical filtered baths (Fig. 1(c)), and given by

$$
A_{c}^{-}:=\zeta_{1}^{2} \tilde{G}_{H}\left(-\omega_{-}\right)\left\langle\tilde{n}_{a}+1\right\rangle, \quad A_{c}^{+}:=\zeta_{1}^{2} \tilde{G}_{H}\left(\omega_{-}\right)\left\langle\tilde{n}_{a}\right\rangle .
$$

The steady-state average phonon number is

$$
\left\langle\tilde{n}_{1}\right\rangle_{\mathrm{ss}}=\frac{A_{c}^{+}+\kappa_{1} \bar{n}_{1}}{\tilde{\Gamma}_{c}+\kappa_{1}}
$$

here $\tilde{\Gamma}_{c}=A_{c}^{-}-A_{c}^{+}$is the net optical baths damping rate. This equation of phonon-number is similar to the steadystate phonon number in a standard optomechanical system for laser sideband cooling [7], which is given by $\left\langle n_{1}\right\rangle_{s s}=$ $\left(A^{+}+\kappa_{1} \bar{n}_{1}\right) /\left(\Gamma_{\mathrm{opt}}+\kappa_{1}\right) . \Gamma_{\mathrm{opt}}=A^{-}-A^{+}$is the net optomechanical damping rate, further, $A^{+}$and $A^{-}$represent the rates of Stokes (heating) and anti-Stokes (cooling) processes, respectively. For the resolved-sideband ground-state laser cooling, (i) upper sideband should be far off-resonant, (ii) the optical damping $\Gamma_{\text {opt }}$ must be much greater than the

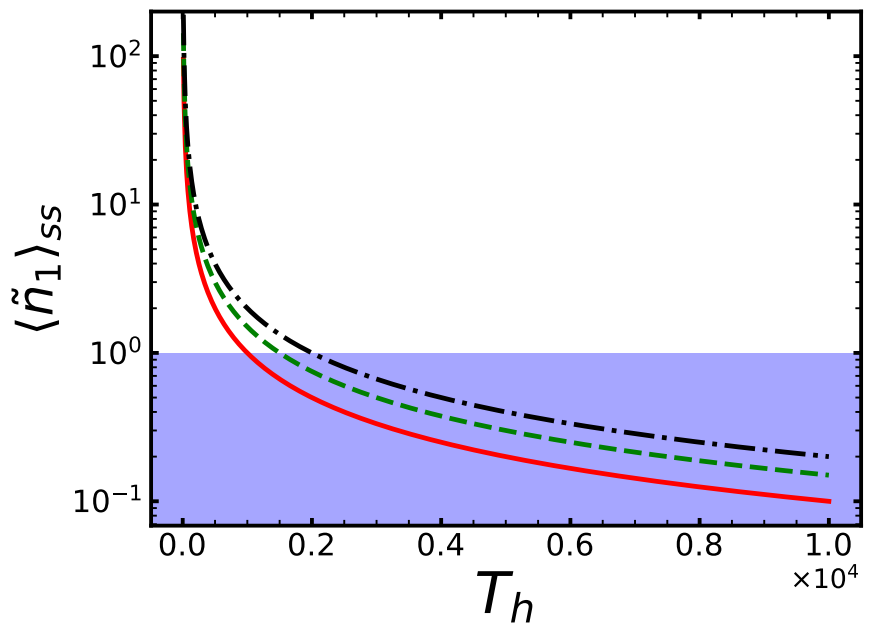

FIG. 3. (Color online) The steady-state mean phonon number $\left\langle\tilde{n}_{1}\right\rangle_{s s}$ as a function of the hot bath temperature $T_{h}$. Solid, dashed, and dot-dashed lines are for $T_{1}=1 \times 10^{-4}, 2 \times 10^{-4}$ and $3 \times 10^{-4}$, respectively. The other parameters are $\omega_{a}=1, \omega_{1}=1 \times 10^{-7}$, $\kappa_{h}=\kappa_{c}=1 \times 10^{-8}, \kappa_{1}=1 \times 10^{-12}, T_{c}=1 \times 10^{-5}$, and $g_{1}=$ $1 \times 10^{-9}$. All the parameters are scaled with the optical frequency $\omega_{a}=2 \pi \times 10^{14} \mathrm{~Hz}$. The temperatures in SI units are given as $T_{c} \approx 5 \mathrm{mK}, T_{1} \approx 0.5,1$ and $1.5 \mathrm{~K}$ for solid, dashed, dot-dashed lines, respectively. The hot bath temperature is higher by many order of magnitude and it is approximately $4 \times 10^{4} \lesssim T_{h} \lesssim 4 \times 10^{7} \mathrm{~K}$.

mechanical damping $\kappa_{1}$, and (iii) the initial phonon number $\bar{n}_{1}(0)$ must be much smaller than the quality factor of the mechanical resonator $\bar{n}_{1} \ll \omega_{1} / \kappa_{1}$ [7]. Similar to the resolvedsideband cooling, in our scheme ground-state cooling is possible provided, (i) upper sideband $\left(\omega_{+}\right)$from the hot bath spectrum is filtered, (ii) for sufficiently low cold bath temperature, the thermal dissipation due to the optical baths is greater than the mechanical bath coupling strength, i.e., $\tilde{\Gamma}_{c} \gg \kappa_{1}$. (iii) The initial phonon number in the mechanical resonator is much smaller than its quality factor $\bar{n}_{1} \ll \omega_{1} / \kappa_{1}$, in addition, we note that these conditions are stated for $T_{h}>T_{1}>T_{c}$. For the bath spectra shown in Fig.1(c), and considered system parameters (Fig. 3), $\tilde{\Gamma}_{c}$ is larger than $\kappa_{1}$ by three order of magnitude. The other conditions mentioned here also satisfy with in the considered system parameters. To investigate the steadystate cooling performance of our scheme, we plot the average number of phonons $\left\langle\tilde{n}_{1}\right\rangle_{s s}$ in the mechanical resonator as a function of the hot bath temperature $T_{h}$ in Fig. 3 . The results indicate that ground-state cooling is possible for the reasonable hot bath temperature.

The cooling mechanism can be explained by direct analogy with the laser sideband cooling shown in Fig. 2. By increasing the temperature $T_{h}$ of the hot bath, the number of photons of "correct frequency" in the cavity to induce the red (lower) sideband transition increases. In a refrigeration process, the hot bath induces a transition of frequency $\omega_{-}$at the cost of destroying a single phonon (Fig. 2). Then the energetic (blue-shifted) photon created in previous transition is dumped in to the cold bath to complete the refrigeration process. Note 
that, this explanation is only valid for the filtered baths spectra shown in Fig. 1(c). For baths spectra including upper phonon sideband or spectrally overlapping baths, this explanation is invalid.

Heating: if we select the optical baths spectra of the form shown in Fig. 1(d), and they follow

$$
\begin{aligned}
\tilde{G}_{C}\left(\omega_{a}\right) & \gg \tilde{G}_{H}\left(\omega_{a}\right), \\
\tilde{G}_{C}\left(\omega_{+}\right) & \ll \tilde{G}_{H}\left(\omega_{+}\right),
\end{aligned}
$$

then the master equation given in Eq. (9) simplifies to

$$
\begin{aligned}
& \tilde{\mathcal{L}}_{C}=\tilde{G}_{C}\left(\omega_{a}\right) \tilde{\mathcal{D}}[\tilde{a}]+\tilde{G}_{C}\left(-\omega_{a}\right) \tilde{\mathcal{D}}\left[\tilde{a}^{\dagger}\right], \\
& \tilde{\mathcal{L}}_{H}=\zeta_{1}^{2}\left(\tilde{G}_{H}\left(\omega_{+}\right) \tilde{\mathcal{D}}\left[\tilde{a} \tilde{b}_{1}\right]+\tilde{G}_{H}\left(-\omega_{+}\right) \tilde{\mathcal{D}}\left[\tilde{a}^{\dagger} \tilde{b}_{1}^{\dagger}\right]\right),
\end{aligned}
$$

and $\tilde{\mathcal{L}}_{1}$ remains the same. The energy transitions induced by these dissipators $\left(\tilde{\mathcal{D}}\left[\tilde{a}^{\dagger} \tilde{b}^{\dagger}\right], \tilde{\mathcal{D}}[\tilde{a} \tilde{b}]\right)$ lead to heating of the mechanical resonator as shown in Fig. 2. $\Gamma_{h}=A_{h}^{-}-A_{h}^{+}$is the net optical thermal dissipation rate with the downward and upward transition rates

$$
A_{h}^{-}:=\zeta_{1}^{2} \tilde{G}_{H}\left(\omega_{+}\right)\left\langle\tilde{n}_{a}\right\rangle, \quad A_{h}^{+}:=\zeta_{1}^{2} \tilde{G}_{H}\left(-\omega_{+}\right)\left\langle\tilde{n}_{a}+1\right\rangle .
$$

The dynamical evolution of the initial phonon number is given by

$$
\left\langle\tilde{n}_{1}(t)\right\rangle=\frac{A_{h}^{+}+\kappa_{1} \bar{n}_{1}}{\tilde{\Gamma}_{h}+\kappa_{1}}+\bar{n}_{1} e^{-t\left(\tilde{\Gamma}_{h}+\kappa_{1}\right)},
$$

in this case, $\tilde{\Gamma}_{h}$ is negative, consequently, the mechanical resonator heats up and the system becomes unstable in the longtime limit.

\section{B. Multiple mechanical resonators}

Here, we consider two mechanical resonators couple to a single optical cavity, as shown in Fig. 1, however, the extension of our scheme to $\mathrm{N}$ mechanical resonators is straightforward. For the case of the hot and cold baths spectra considered in Fig. 1(c), the reduced master equation of the two mechanical resonators simplifies to

$$
\frac{d \tilde{\rho}}{d t}=\left(A_{i}^{-}+A_{\mathrm{th}, i}^{-}\right) \tilde{\mathcal{D}}\left[\tilde{b}_{i}\right]+\left(A_{i}^{+}+A_{\mathrm{th}, i}^{+}\right) \tilde{\mathcal{D}}\left[\tilde{b}_{i}^{\dagger}\right]
$$

and the transitions rates due to the optical baths have the form $A_{i}^{-}:=\zeta_{i}^{2} \tilde{G}_{H}\left(-\omega_{-, i}\right)\left\langle\tilde{n}_{a}+1\right\rangle, \quad A_{i}^{+}:=\zeta_{i}^{2} \tilde{G}_{H}\left(\omega_{-, i}\right)\left\langle\tilde{n}_{a}\right\rangle$, $\omega_{-, i}=\omega_{a}-\omega_{i}$. At steady-state the average phonon number in each resonator is given by

$$
\left\langle\tilde{n}_{i}\right\rangle_{\mathrm{ss}}=\frac{A_{i}^{+}+\kappa_{i} \bar{n}_{i}}{\tilde{\Gamma}_{i}+\kappa_{i}},
$$

here $\tilde{\Gamma}_{i}=A_{i}^{-}-A_{i}^{+}$. This shows that the steady-state mean phonon number of each resonator depends on the relaxation rates of both resonators with their baths. The simultaneous cooling of both degenerate and non-degenerate multiple resonators is possible provided these relaxation rates are not
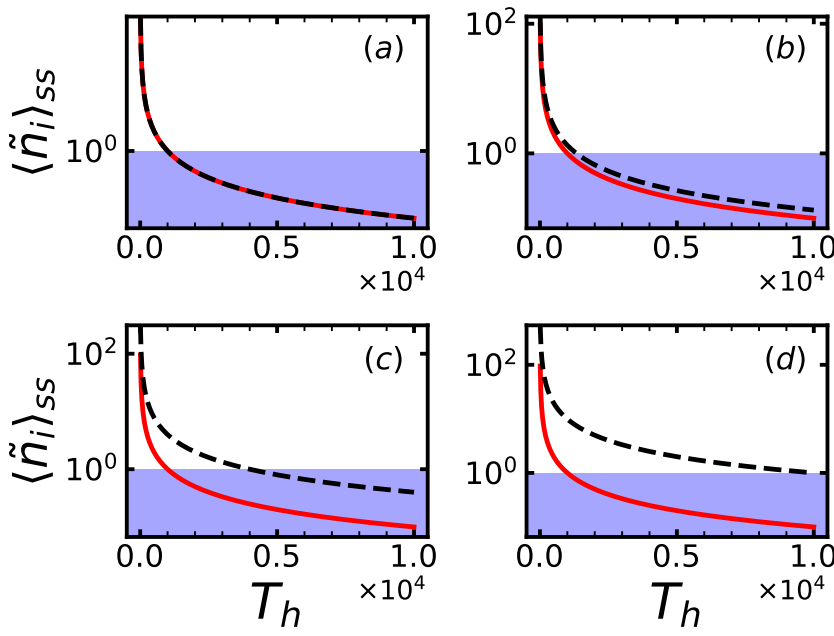

FIG. 4. (Color online) The steady-state mean phonon number $\left\langle\tilde{n}_{i}\right\rangle_{s s}$ as a function of hot bath temperature $T_{h}$. Solid, and dashed lines are for the first and second mechanical resonator, respectively. Parameters: $T_{1}=T_{2}=2 \times 10^{-4}$, other parameters are the same as in Fig. 3. (a) $\omega_{1}=\omega_{2}, g_{1}=g_{2}$, (b) $\omega_{2}=0.75 \omega_{1}, g_{1}=g_{2}$, (c) $g_{2}=0.5 g_{1}, \omega_{1}=\omega_{2}$, and (d) $\kappa_{2}=10 \kappa_{1}, \omega_{1}=\omega_{2}, g_{1}=g_{2}$.

very high. On contrary, in optomechanical sideband cooling, existence of the dark modes hinder the simultaneous multiple resonators cooling [31]. In addition, for degenerate or nearly degenerate resonators, one can not cool down either of the mechanical resonators [18, 30]. In our scheme, Eq. (29) shows that, simultaneous cooling of both degenerate and nondegenerate resonators is possible. This is shown in Fig. 4, in which we plot the steady-state phonon number $\left\langle\tilde{n}_{i}\right\rangle_{s S}$ of each resonator as a function of the hot bath temperature $T_{h}$. For the identical resonators (Fig. 4(a)) coupled to thermal baths of the same temperature, the cooling curves are identical. However, for the non-degenerate case (Fig. 4(b)), the steady-state phonons in the second resonator is greater than the first resonator. The lesser cooling of the second resonator is because of the choice of the hot bath spectrum (Fig. 1(c)), which peaks at $\omega_{a}-\omega_{1}$. The second resonator frequency $\omega_{2}$ is below this peak, due to which it is weakly driven as compared to the resonator one, consequently, $\tilde{\Gamma}_{2}<\tilde{\Gamma}_{1}$. The effect of optomechanical coupling strength $g_{i}$ is shown in Fig. 4(c), the weakly coupled second resonator have smaller optical thermal dissipation $\left(\tilde{\Gamma}_{2}<\tilde{\Gamma}_{1}\right)$, which results in lesser cooling. The mechanical resonator with larger system-bath coupling cools lesser as shown in Fig. 4(d).

Extension of our scheme to $\mathrm{N}$ independent mechanical resonators coupled to a single cavity mode is straightforward. Because Eqs. (28) and (29) remain valid for arbitrary number of independent resonators. Another key feature of our method is that it is not only limited to optomechanics, but can also be realized in different platforms provided the target system to be cooled interacts dispersively with the working medium through the Hamiltonian of the form $\hat{H}_{\text {int }}=g N_{o} X_{m}$. Where $X_{m}$ is observable of the target system, and $N_{o}=\eta \hat{H}_{\mathrm{wm}}$ with $\eta$ a positive constant, such an interaction yields a master equa- 


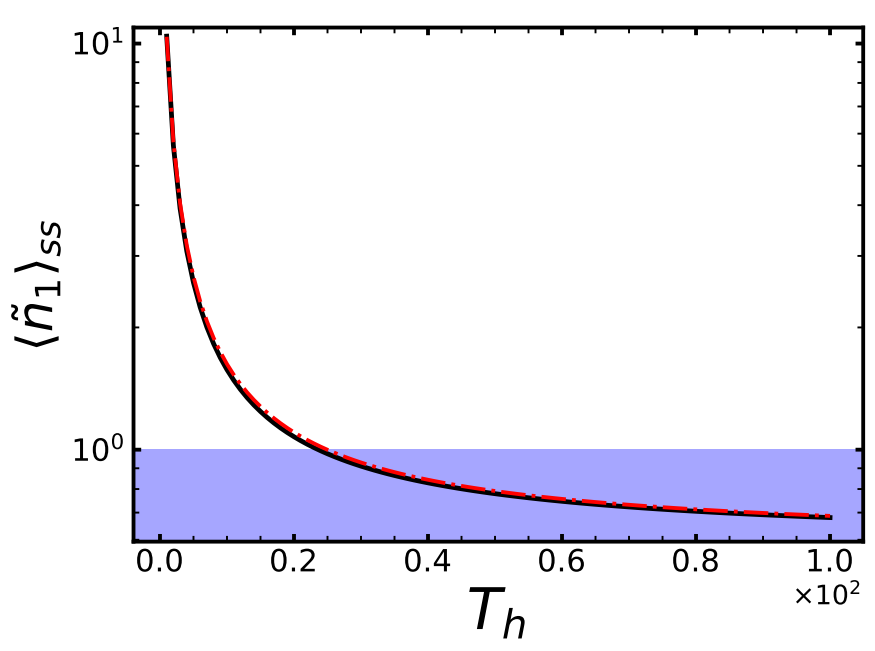

FIG. 5. (Color online) The steady-state mean phonon number $\left\langle\tilde{n}_{1}\right\rangle_{s s}$ as a function of the hot bath temperature $T_{h}$. Solid line is for Eq. (23) in case of ignoring $\zeta_{1}^{2}$ terms from $\left\langle\tilde{n}_{a}\right\rangle_{s s}$ (Eq. (21)), and dot-dashed line represents $\left\langle\tilde{n}_{1}\right\rangle_{s s}$ calculated by the numerical simulation of the master equation given in Eq. (20). In the numerical simulation, we use truncated Hilbert space with 7 photon states and 70 phonon states. The use of small Hilbert space dimensions for the optical mode is justified, because $\left\langle\tilde{n}_{a}\right\rangle_{\text {ss }} \ll 1$ for all parameters considered. We have verified that the increase in the Hilbert space dimensions does not affect the results. Parameters: $\bar{n}_{1}=10, \bar{n}_{c}=0.5$, and rest of the parameters are same as given in Fig. 3.

tion of the form given in Eq. (9). For example, a qubit dispersively coupled to a superconducting resonator [39, 51], or with a nanomechanical resonator [52].

\section{CONCLUSIONS}

We proposed a scheme to implement ground-state cooling of a single mechanical resonator in the standard optomechanical setting. As opposed to laser cooling, we used hot thermal light to cool down the mechanical resonator. We showed that it is possible to cool multiple degenerate or near-degenerate mechanical resonators to their ground-state using our method. To realize our proposed scheme, we used bath spectrum filtering to remove the unwanted transition frequencies from the system-bath coupling spectra. Remarkably, the steadystate mean phonon number given in Eq. (23) shares similarities with the average phonon number obtained in laser sideband cooling. Consequently, ground-state cooling conditions in our scheme are also similar to the those given in standard resolved-sideband cooling. Temperatures for the cavity field are most useful for cooling when they are comparable or hotter than the solar temperatures $\sim 5000 \mathrm{~K}$. Intriguingly, the mechanical components would not equilibrate with such a high temperature, which would melt them, but thermalize to a much colder temperature. An appealing possibility to get high temperatures for the cavity field could be to use micromaser scheme of heating [53], where the pump atoms can be prepared in quantum coherent superpositions [54, 55]. Instead of a beam of atoms, one can also consider using a single atom making repeated interactions with the cavity field. By this way, one can cool multiple mechanical resonators using even a single non-thermal atom carrying quantum information [56].

Our scheme of cooling establishes a direction connection between sideband cooling and cooling by heating methods proposed previously $[35,36]$. These results are relevant to the miniaturization of quantum devices based on the novel multimode optomechanical systems [28, 57].

\section{Appendix A: Dynamics of the system}

For the optomechanical system shown in Fig. 1(a), if we consider a single mechanical resonator of frequency $\omega_{1}$, and bath spectrum filtering of Fig. 1(c), the master equation can be written as [58]

$$
\begin{aligned}
\tilde{\mathcal{L}}_{C} & =\gamma_{c}\left(\tilde{\mathcal{D}}[\tilde{a}]+e^{-\beta_{c} \omega_{a}} \tilde{\mathcal{D}}\left[\tilde{a}^{\dagger}\right]\right) \\
\tilde{\mathcal{L}}_{H} & =\gamma_{h} \zeta_{1}^{2}\left(\tilde{\mathcal{D}}\left[\tilde{a} \tilde{b}_{1}^{\dagger}\right]+e^{-\beta_{h} \omega_{+}} \tilde{\mathcal{D}}\left[\tilde{a}^{\dagger} \tilde{b}_{1}\right]\right), \\
\tilde{\mathcal{L}}_{1} & =\gamma_{1}\left(\tilde{\mathcal{D}}\left[\tilde{b}_{1}\right]+e^{-\beta_{1} \omega_{1}} \tilde{\mathcal{D}}\left[\tilde{b}_{1}^{\dagger}\right]\right),
\end{aligned}
$$

here $\gamma_{c}, \gamma_{h}$, and $\gamma_{1}$ are relaxation rates which depend on the specific model of the cold, hot and mechanical baths, respectively. In addition, $\beta_{c}, \beta_{h}$, and $\beta_{1}$ are inverse temperatures of the cold, hot and mechanical baths, respectively. In the limit $\beta_{h} \rightarrow 0$, the rate equations for the mean number of photons and phonons read

$$
\begin{aligned}
\frac{d}{d t}\left\langle\tilde{n}_{a}\right\rangle= & -\gamma_{c}\left(1-e^{-\beta_{c} \omega_{a}}\right)\left\langle\tilde{n}_{a}\right\rangle+\gamma_{c} e^{-\beta_{c} \omega_{a}} \\
& +\gamma_{h}\left(\left\langle\tilde{n}_{1}\right\rangle-\left\langle\tilde{n}_{a}\right\rangle\right), \\
\frac{d}{d t}\left\langle\tilde{n}_{1}\right\rangle= & -\gamma_{1}\left(1-e^{-\beta_{1} \omega_{1}}\right)\left\langle\tilde{n}_{1}\right\rangle+\gamma_{1} e^{-\beta_{1} \omega_{1}} \\
& +\gamma_{h}\left(\left\langle\tilde{n}_{a}\right\rangle-\left\langle\tilde{n}_{1}\right\rangle\right),
\end{aligned}
$$

and at steady-state the mean number of quanta in the mechanical resonator take the form

$$
\left\langle\tilde{n}_{1}\right\rangle_{\mathrm{ss}}=\frac{\gamma_{c} e^{-\beta_{c} \omega_{a}}+\gamma_{1} e^{-\beta_{1} \omega_{1}}}{-\gamma_{c} e^{-\beta_{c} \omega_{a}}-\gamma_{1} e^{-\beta_{1} \omega_{1}}+\gamma_{c}+\gamma_{1}} .
$$

For one-dimensional Ohmic spectral densities of the baths, Eq. (A6) simplifies to

$$
\left\langle\tilde{n}_{1}\right\rangle_{\mathrm{ss}}=\frac{\omega_{a} \kappa_{c} \bar{n}_{c}+\omega_{1} \kappa_{1} \bar{n}_{1}}{\omega_{a} \kappa_{c}+\omega_{1} \kappa_{1}},
$$

which shows that ground-state cooling is possible for the conditions stated in Sec. III A. 
[1] Alex Abramovici, William E. Althouse, Ronald W. P. Drever, Yekta Gürsel, Seiji Kawamura, Frederick J. Raab, David Shoemaker, Lisa Sievers, Robert E. Spero, Kip S. Thorne, Rochus E. Vogt, Rainer Weiss, Stanley E. Whitcomb, and Michael E. Zucker, "Ligo: The laser interferometer gravitational-wave observatory," Science 256, 325-333 (1992).

[2] M. D. LaHaye, O. Buu, B. Camarota, and K. C. Schwab, "Approaching the quantum limit of a nanomechanical resonator," Science 304, 74-77 (2004).

[3] William Marshall, Christoph Simon, Roger Penrose, and Dik Bouwmeester, "Towards quantum superpositions of a mirror," Phys. Rev. Lett. 91, 130401 (2003).

[4] Stefano Mancini, David Vitali, and Paolo Tombesi, "Scheme for teleportation of quantum states onto a mechanical resonator," Phys. Rev. Lett. 90, 137901 (2003).

[5] D. Vitali, S. Gigan, A. Ferreira, H. R. Böhm, P. Tombesi, A. Guerreiro, V. Vedral, A. Zeilinger, and M. Aspelmeyer, "Optomechanical entanglement between a movable mirror and a cavity field," Phys. Rev. Lett. 98, 030405 (2007).

[6] I. Wilson-Rae, N. Nooshi, W. Zwerger, and T. J. Kippenberg, "Theory of ground state cooling of a mechanical oscillator using dynamical backaction," Phys. Rev. Lett. 99, 093901 (2007).

[7] Florian Marquardt, Joe P. Chen, A. A. Clerk, and S. M. Girvin, "Quantum theory of cavity-assisted sideband cooling of mechanical motion,” Phys. Rev. Lett. 99, 093902 (2007).

[8] K. R. Brown, J. Britton, R. J. Epstein, J. Chiaverini, D. Leibfried, and D. J. Wineland, "Passive cooling of a micromechanical oscillator with a resonant electric circuit," Phys. Rev. Lett. 99, 137205 (2007).

[9] C. Genes, D. Vitali, P. Tombesi, S. Gigan, and M. Aspelmeyer, "Ground-state cooling of a micromechanical oscillator: Comparing cold damping and cavity-assisted cooling schemes," Phys. Rev. A 77, 033804 (2008).

[10] A. Schliesser, R. Rivière, G. Anetsberger, O. Arcizet, and T. J. Kippenberg, "Resolved-sideband cooling of a micromechanical oscillator," Nat. Phys. 4, 415-419 (2008).

[11] C. Genes, D. Vitali, P. Tombesi, S. Gigan, and M. Aspelmeyer, "Ground-state cooling of a micromechanical oscillator: Comparing cold damping and cavity-assisted cooling schemes," Phys. Rev. A 79, 039903 (2009).

[12] J. D. Teufel, T. Donner, Dale Li, J. W. Harlow, M. S. Allman, K. Cicak, A. J. Sirois, J. D. Whittaker, K. W. Lehnert, and R. W. Simmonds, "Sideband cooling of micromechanical motion to the quantum ground state," Nature 475, 359-363 (2011).

[13] Xunnong Xu, Thomas Purdy, and Jacob M. Taylor, "Cooling a harmonic oscillator by optomechanical modification of its bath," Phys. Rev. Lett. 118, 223602 (2017).

[14] P. F. Cohadon, A. Heidmann, and M. Pinard, "Cooling of a mirror by radiation pressure," Phys. Rev. Lett. 83, 3174-3177 (1999).

[15] Dustin Kleckner and Dirk Bouwmeester, "Sub-kelvin optical cooling of a micromechanical resonator," Nature 444, 75-78 (2006).

[16] Thomas Corbitt, Christopher Wipf, Timothy Bodiya, David Ottaway, Daniel Sigg, Nicolas Smith, Stanley Whitcomb, and Nergis Mavalvala, "Optical dilution and feedback cooling of a gram-scale oscillator to 6.9 mk," Phys. Rev. Lett. 99, 160801 (2007).

[17] M. Poggio, C. L. Degen, H. J. Mamin, and D. Rugar, "Feedback cooling of a cantilever's fundamental mode below $5 \mathrm{mk}$," Phys. Rev. Lett. 99, 017201 (2007).
[18] Christian Sommer and Claudiu Genes, "Partial optomechanical refrigeration via multimode cold-damping feedback," Phys. Rev. Lett. 123, 203605 (2019).

[19] Christian Sommer, Alekhya Ghosh, and Claudiu Genes, "Multimode cold-damping optomechanics with delayed feedback," Phys. Rev. Research 2, 033299 (2020).

[20] A. Schliesser, P. Del'Haye, N. Nooshi, K. J. Vahala, and T. J. Kippenberg, "Radiation pressure cooling of a micromechanical oscillator using dynamical backaction,” Phys. Rev. Lett. 97, 243905 (2006).

[21] R. W. Peterson, T. P. Purdy, N. S. Kampel, R. W. Andrews, P.L. Yu, K. W. Lehnert, and C. A. Regal, "Laser cooling of a micromechanical membrane to the quantum backaction limit," Phys. Rev. Lett. 116, 063601 (2016).

[22] Juan Pablo Paz and Augusto J. Roncaglia, "Dynamics of the entanglement between two oscillators in the same environment," Phys. Rev. Lett. 100, 220401 (2008).

[23] Ying-Dan Wang and Aashish A. Clerk, "Reservoir-engineered entanglement in optomechanical systems," Phys. Rev. Lett. 110, 253601 (2013).

[24] A. Mari, A. Farace, N. Didier, V. Giovannetti, and R. Fazio, "Measures of quantum synchronization in continuous variable systems," Phys. Rev. Lett. 111, 103605 (2013).

[25] M. J. Woolley and A. A. Clerk, "Two-mode squeezed states in cavity optomechanics via engineering of a single reservoir," Phys. Rev. A 89, 063805 (2014).

[26] Matthew H. Matheny, Matt Grau, Luis G. Villanueva, Rassul B. Karabalin, M. C. Cross, and Michael L. Roukes, "Phase synchronization of two anharmonic nanomechanical oscillators," Phys. Rev. Lett. 112, 014101 (2014).

[27] André Xuereb, Alberto Imparato, and Aurélien Dantan, "Heat transport in harmonic oscillator systems with thermal baths: application to optomechanical arrays," New J. Phys. 17, 055013 (2015).

[28] Shabir Barzanjeh, Matteo Aquilina, and André Xuereb, "Manipulating the flow of thermal noise in quantum devices," Phys. Rev. Lett. 120, 060601 (2018).

[29] C. F. Ockeloen-Korppi, E. Damskägg, J.-M. Pirkkalainen, M. Asjad, A. A. Clerk, F. Massel, M. J. Woolley, and M. A. Sillanpää, "Stabilized entanglement of massive mechanical oscillators," Nature 556, 478-482 (2018).

[30] Claudiu Genes, David Vitali, and Paolo Tombesi, "Simultaneous cooling and entanglement of mechanical modes of a micromirror in an optical cavity," New J. Phys. 10, 095009 (2008).

[31] C. F. Ockeloen-Korppi, M. F. Gely, E. Damskägg, M. Jenkins, G. A. Steele, and M. A. Sillanpää, "Sideband cooling of nearly degenerate micromechanical oscillators in a multimode optomechanical system," Phys. Rev. A 99, 023826 (2019).

[32] A. B. Shkarin, N. E. Flowers-Jacobs, S. W. Hoch, A. D. Kashkanova, C. Deutsch, J. Reichel, and J. G. E. Harris, "Optically mediated hybridization between two mechanical modes," Phys. Rev. Lett. 112, 013602 (2014).

[33] Deng-Gao Lai, Fen Zou, Bang-Pin Hou, Yun-Feng Xiao, and Jie-Qiao Liao, "Simultaneous cooling of coupled mechanical resonators in cavity optomechanics," Phys. Rev. A 98, 023860 (2018).

[34] Deng-Gao Lai, Jin-Feng Huang, Xian-Li Yin, Bang-Pin Hou, Wenlin Li, David Vitali, Franco Nori, and Jie-Qiao Liao, "Nonreciprocal ground-state cooling of multiple mechanical resonators," Phys. Rev. A 102, 011502 (2020).

[35] A. Mari and J. Eisert, "Cooling by heating: Very hot thermal 
light can significantly cool quantum systems," Phys. Rev. Lett. 108, 120602 (2012).

[36] B. Cleuren, B. Rutten, and C. Van den Broeck, "Cooling by heating: Refrigeration powered by photons," Phys. Rev. Lett. 108, 120603 (2012).

[37] A.G. Kofman, G. Kurizki, and B. Sherman, "Spontaneous and induced atomic decay in photonic band structures," J. Mod. Opt. 41, 353-384 (1994).

[38] D. Gelbwaser-Klimovsky, R. Alicki, and G. Kurizki, "Minimal universal quantum heat machine," Phys. Rev. E 87, 012140 (2013).

[39] D. Gelbwaser-Klimovsky and G. Kurizki, "Heat-machine control by quantum-state preparation: From quantum engines to refrigerators," Phys. Rev. E 90, 022102 (2014).

[40] D. Gelbwaser-Klimovsky and G. Kurizki, "Work extraction from heat-powered quantized optomechanical setups," Sci. Rep. 5, 7809 (2015).

[41] A. Ghosh, C. L. Latune, L. Davidovich, and G. Kurizki, "Catalysis of heat-to-work conversion in quantum machines," Proc. Natl. Acad. Sci. 114, 12156-12161 (2017).

[42] Arnab Ghosh, David Gelbwaser-Klimovsky, Wolfgang Niedenzu, Alexander I. Lvovsky, Igor Mazets, Marlan O. Scully, and Gershon Kurizki, "Two-level masers as heat-towork converters," Proc. Natl. Acad. of Sci. 115, 9941-9944 (2018).

[43] M Tahir Naseem, Avijit Misra, and Özgür E. Müstecaplioğlu, "Two-body quantum absorption refrigerators with optomechanical-like interactions," Quantum Sci. Technol. 5, 035006 (2020).

[44] M. Tahir Naseem, Avijit Misra, Özgür E. Müstecaplioğlu, and Gershon Kurizki, "Minimal quantum heat manager boosted by bath spectral filtering," Phys. Rev. Research 2, 033285 (2020).

[45] Kejie Fang, Jie Luo, Anja Metelmann, Matthew H. Matheny, Florian Marquardt, Aashish A. Clerk, and Oskar Painter, "Generalized non-reciprocity in an optomechanical circuit via synthetic magnetism and reservoir engineering," Nat. Phys. 13, 465-471 (2017).

[46] F. Massel, T. T. Heikkilä, J.-M. Pirkkalainen, S. U. Cho, H. Saloniemi, P. J. Hakonen, and M. A. Sillanpää, "Microwave amplification with nanomechanical resonators," Nature 480, 351-
354 (2011).

[47] Francesco Massel, Sung Un Cho, Juha-Matti Pirkkalainen, Pertti J. Hakonen, Tero T. Heikkilä, and Mika A. Sillanpää, "Multimode circuit optomechanics near the quantum limit," Nat. Commun. 3, 987 (2012).

[48] M. Tahir Naseem, André Xuereb, and Özgür E. Müstecaplıoğlu, "Thermodynamic consistency of the optomechanical master equation," Phys. Rev. A 98, 052123 (2018).

[49] Markus Aspelmeyer, Tobias J. Kippenberg, and Florian Marquardt, "Cavity optomechanics," Rev. Mod. Phys. 86, 13911452 (2014).

[50] C. L. Latune, I. Sinayskiy, and F. Petruccione, "Quantum coherence, many-body correlations, and non-thermal effects for autonomous thermal machines," Sci. Rep. 9, 3191 (2019).

[51] Ze-Liang Xiang, Sahel Ashhab, J. Q. You, and Franco Nori, "Hybrid quantum circuits: Superconducting circuits interacting with other quantum systems," Rev. Mod. Phys. 85, 623-653 (2013).

[52] A D Armour and M P Blencowe, "Probing the quantum coherence of a nanomechanical resonator using a superconducting qubit: I. echo scheme," New J. Phys. 10, 095004 (2008).

[53] Marlan O. Scully and Willis E. Lamb, "Quantum theory of an optical maser. i. general theory," Phys. Rev. 159, 208-226 (1967).

[54] C. B. Dağ, W. Niedenzu, Özgür E. Müstecaplioğlu, and G. Kurizki, "Multiatom quantum coherences in micromasers as fuel for thermal and nonthermal machines," Entropy 18, 144 (2016).

[55] A. T. Özdemir and Özgür E. Müstecaplioğlu, "Quantum thermodynamics and quantum coherence engines," Turk. J. Phys. 44, 404-436 (2020).

[56] Deniz Türkpençe and Özgür E. Müstecaplığlu, "Quantum fuel with multilevel atomic coherence for ultrahigh specific work in a photonic carnot engine," Phys. Rev. E 93, 012145 (2016).

[57] Alireza Seif, Wade DeGottardi, Keivan Esfarjani, and Mohammad Hafezi, "Thermal management and non-reciprocal control of phonon flow via optomechanics," Nat. Commun. 9, 1207 (2018).

[58] Amikam Levy, Robert Alicki, and Ronnie Kosloff, "Quantum refrigerators and the third law of thermodynamics," Phys. Rev. E 85, 061126 (2012). 
(a)

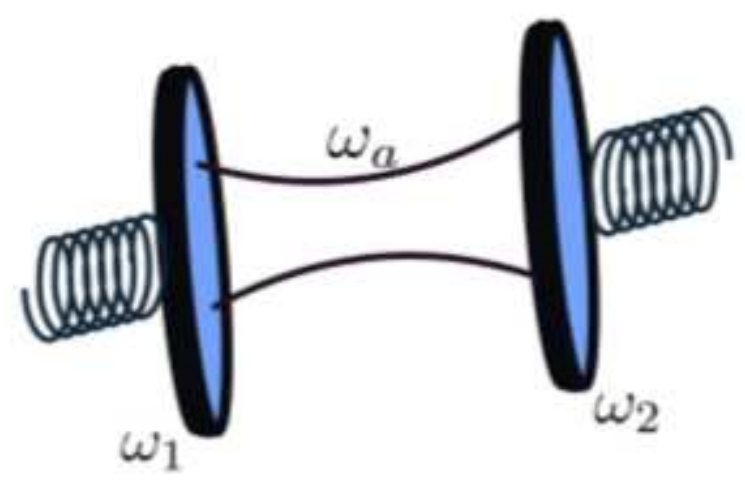

(c)

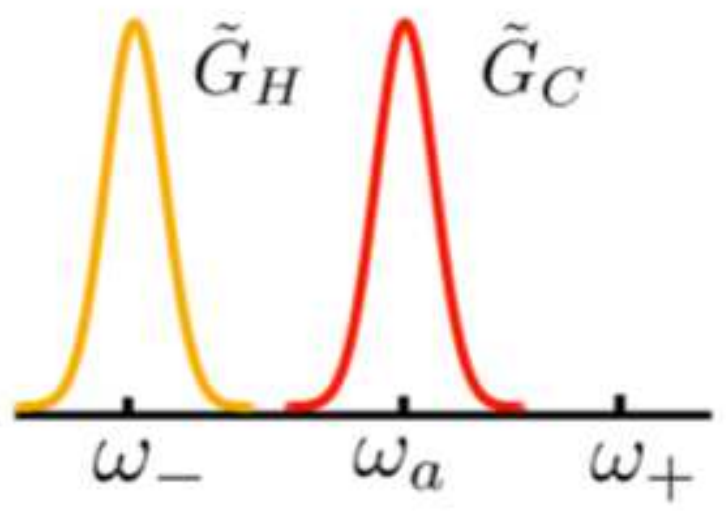

(b)

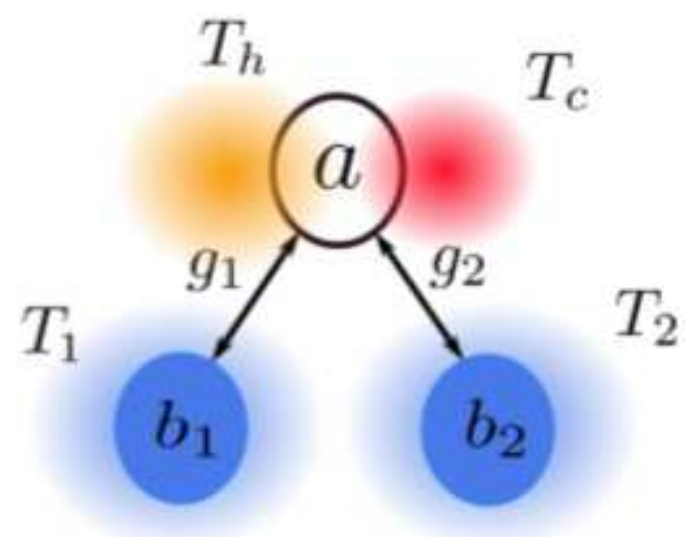

(d)

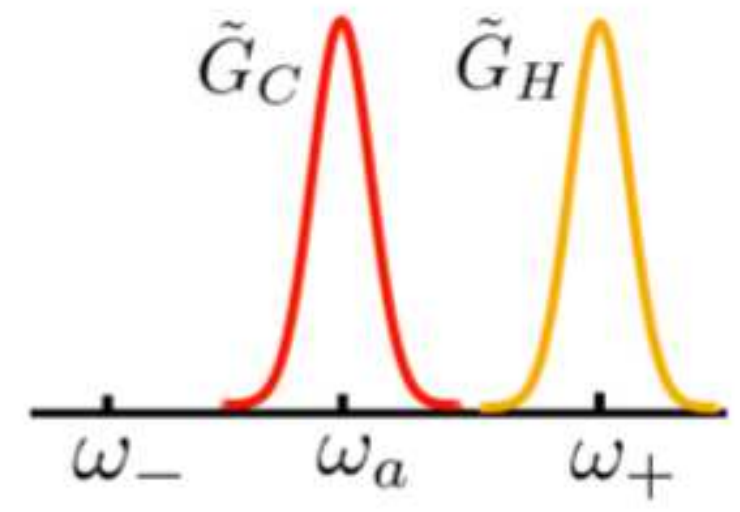

Figure 1

(Color online) (a) Schematic illustration of the optomechanical system being considered in this work. A single optical mode of frequency $\omega a$ iscoupledtotwomechanicalresonatorsoffrequencies $\omega 1$ and $\omega 2$ viaoptomechanicalcouplings g1 and g2,respectively. (b) Each mechanical resonator (bi) of frequency wi is in contact with an independent thermal bath at temperature $T i, i=1,2$. In addition, the optical mode is in contact with two independent thermal baths at temperatures Th and Tc. (c) and (d) filtered spectra of the optical hot and cold baths given by Eq. (18), for cooling and heating process, respectively. 


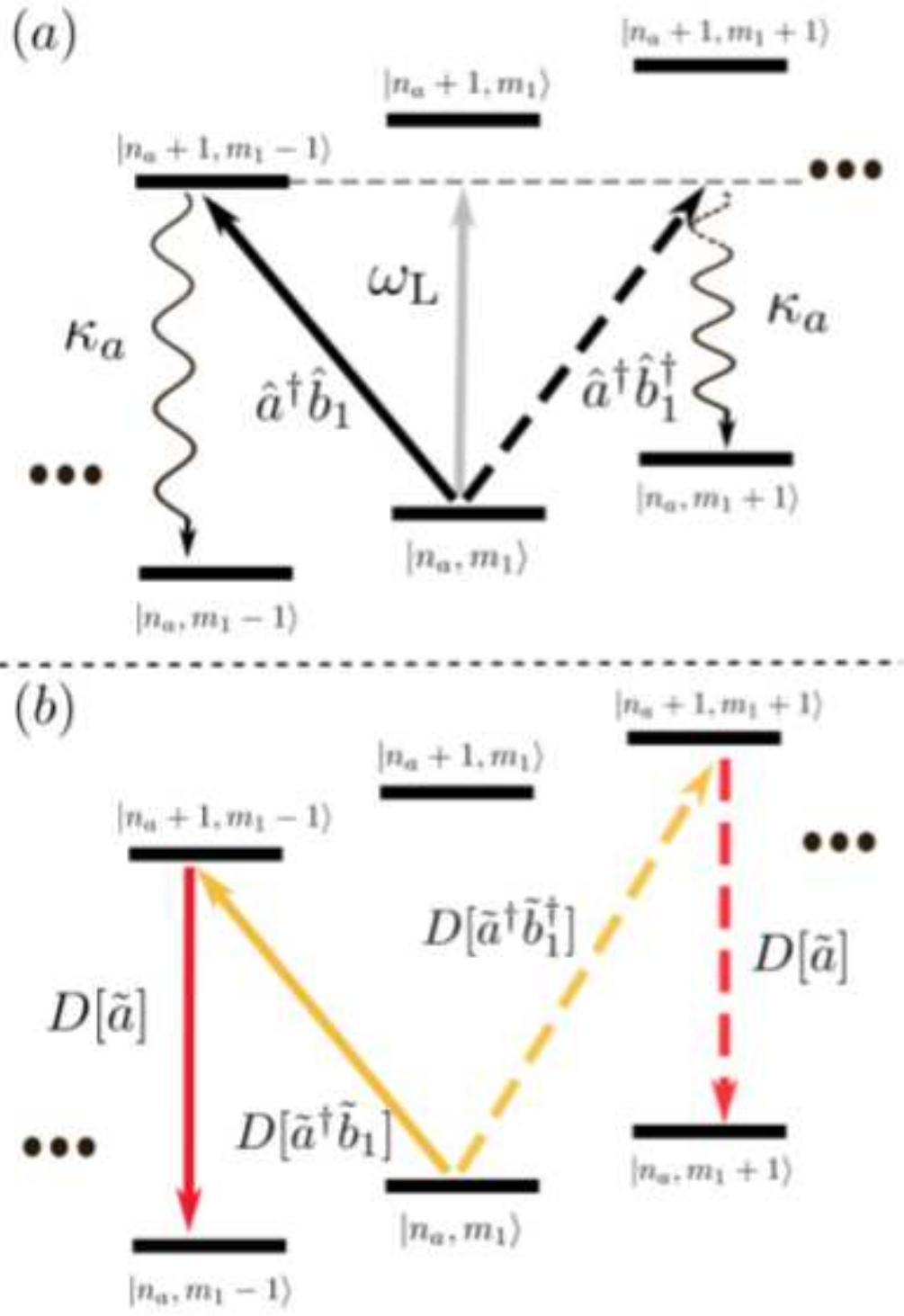

Figure 2

(Color online)The underlying energy transitions between the dressed states Ina,m1 $\mathrm{i}$ of the optomechanical system with a single mechanical resonator. In both panels, solid and dashed lines show the cooling and heating process, respectively. (a) The cooling and heating processes in the presence of coherent laser drive of frequency $\omega L$. The cooling process proceeds by the absorption on the lower sideband, leading to the leakage of a blue-shifted photon from the cavity by destroying one phonon. On contrary, absorption at the upper sideband leads to the heating of the mechanical resonator [10]. (b)The energy transitions involved in the cooling and heating process in our proposed scheme. As opposed to laser cooling, the transition at lower sideband $(\omega-)$ is induced by the hot bath via the incoherent systembath interaction, given by $D[a ̃ ~ \sim b \dagger 1]$ in Eq.(10). This creates an energetic (blue-shifted) photon which is dumped in a bath at lower temperature Tc. Cooling with a single optical bath is not allowed by the second law of thermodyanics. Because, in the case of a single optical bath, $\mid$ na,m1 $1>\nabla \mid$ na +1 , $m 1>1$ i $\mid$ |na,m1 $>1$ itransitions mean heat flows from the mechanical to optical bath for $\mathrm{T} 1<\mathrm{Th}$, hence we need another bath to be consistent with the second law of thermodynamics. In the heating process, the 
hot bath induce the transition of frequency $\omega+$ following by dumping of a photon in the cold bath, which results in heating of the mechanical resonator. For the cooling process to dominate, the upper sideband frequency $\omega+$ is filteredfromthesystem-bathcouplingspectra,asshowninFig.1(c).

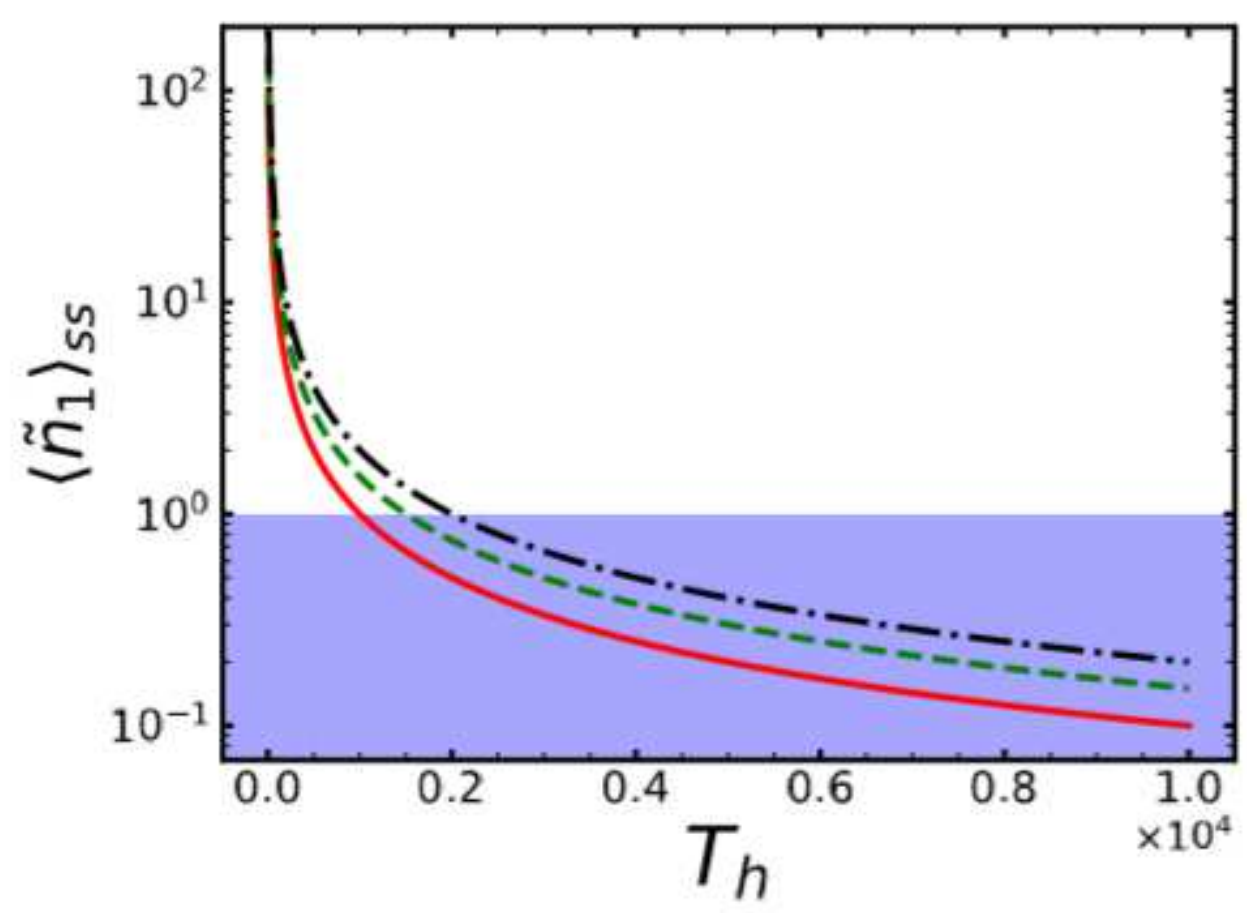

\section{Figure 3}

(Color online)The steady-state mean phonon number $<\tilde{n} 1>$ ss as $s$ a function of the hot bath temperature Th. Solid, dashed, and dot-dashed lines are for T1 $=1 \times 10-4,2 \times 10-4$ and $3 \times 10-4$, respectively. The other parameters are $\omega \mathrm{a}=1, \omega 1=1 \times 10-7, \mathrm{kh}=\mathrm{kc}=1 \times 10-8, \mathrm{k} 1=1 \times 10-12, \mathrm{Tc}=1 \times 10-5$, and g1 $=1 \times 10-9$. All the parameters are scaled with the optical frequency $\omega \mathrm{a}=2 \pi \times 1014 \mathrm{~Hz}$. The temperatures in SI units are given as $\mathrm{Tc} \approx 5 \mathrm{mK}, \mathrm{T} 1 \approx 0.5,1$ and $1.5 \mathrm{~K}$ for solid, dashed, dot-dashed lines, respectively. The hot bath temperature is higher by many order of magnitude and it is approximately $4 \times 104 \sim<\mathrm{Th} \sim<4 \times 107 \mathrm{~K}$. 

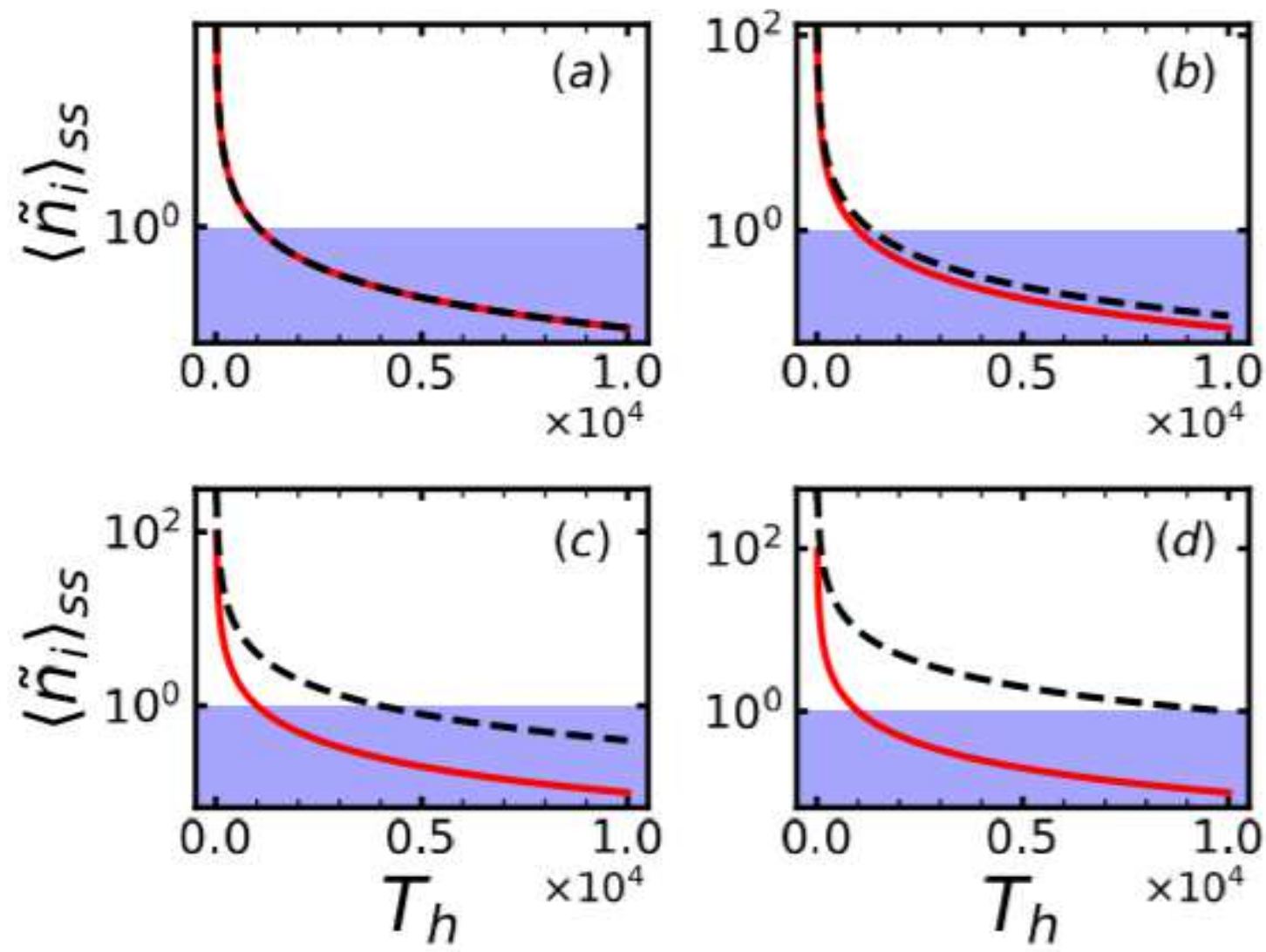

Figure 4

(Color online) The steady-state mean phonon phonon number $<\tilde{n} 1>$ ss a function of hot bath temperature Th. Solid, and dashed lines are for the first and second mechanical resonator, respectively. Parameters: $\mathrm{T} 1=\mathrm{T} 2=2 \times 10-4$, other parameters are the same as in Fig. 3. (a) $\omega 1=\omega 2, \mathrm{~g} 1=\mathrm{g} 2$, (b) $\omega 2=0.75 \omega 1, \mathrm{~g} 1$ $=g 2$, (c) $g 2=0.5 \mathrm{~g} 1, \omega 1=\omega 2$, and (d) $\mathrm{k} 2=10 \mathrm{k} 1, \omega 1=\omega 2, \mathrm{~g} 1=\mathrm{g} 2$. 


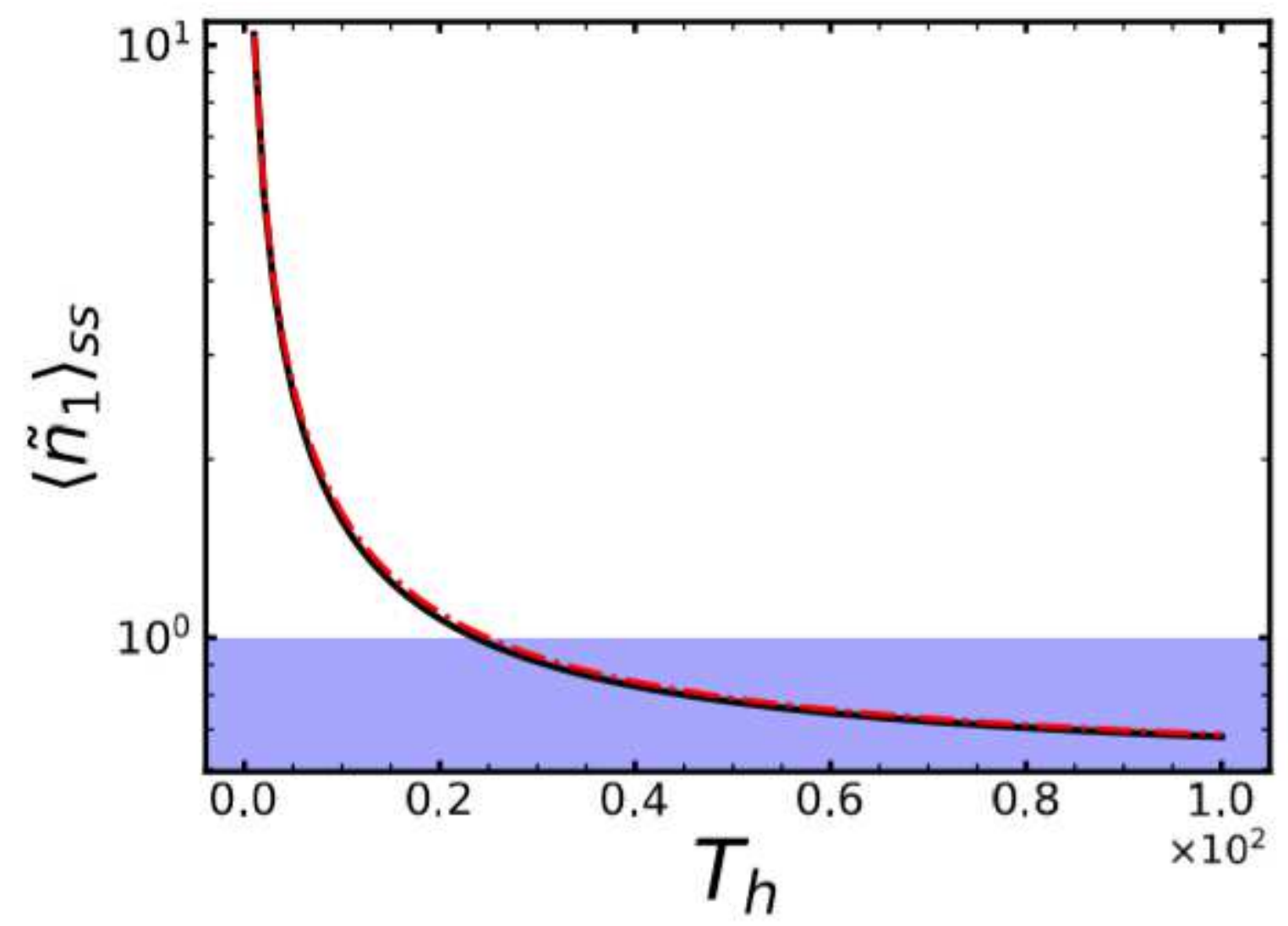

Figure 5

(Color online)The steady-state mean phonon number $<\tilde{n} 1>$ ss as a function of the hot bath temperature Th. Solid line is for Eq.(23) in case of ignoring 721 terms from <ña> ss (Eq. (21)), and dot-dashed line represents $<\tilde{n} 1>$ ss calculated by the numerical simulation of the master equation given in Eq. (20). In the numerical simulation, we use truncated Hilbert space with 7 photon states and 70 phonon states. The use of small Hilbert space dimensions for the optical mode is justified, because $<$ ña $>$ ss $<<1$ for all parameters considered. We have verified that the increase in the Hilbert space dimensions does not affect

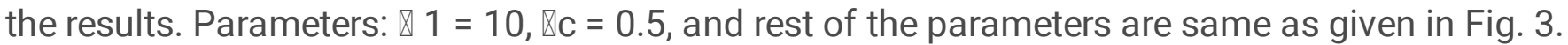

